\title{
ISOENZIMAS NO MONITORAMENTO DA DETERIORAÇÃO DE SEMENTES DE Euterpe espiritosantensis FERNANDES ${ }^{1}$
}

\author{
Cibele Chalita Martins², João Nakagawa ${ }^{3}$ e Paulo Roberto Rodrigues Ramos ${ }^{4}$
}

\begin{abstract}
RESUMO - As sementes de Euterpe espiritosantensis são recalcitrantes, pois apresentam redução da germinação com a desidratação e curta longevidade. O objetivo deste trabalho foi identificar sistemas enzimáticos eficientes no monitoramento da deterioração e perda da capacidade germinativa de sementes de palmiteiro-vermelho. As sementes foram colocadas para secar por 0, 20 e 40 h (teor de água de 46, 40 e 36\%, respectivamente) e armazenadas a $15^{\circ} \mathrm{C}$ em sacos plásticos fechados durante 54 semanas. Em intervalos de tempo de seis semanas, a qualidade das sementes foi avaliada quanto à germinação e atividade das enzimas glucose-6-fosfato desidrogenase, glutamato desidrogenase, fosfoglucomutase, fosfoglucose isomerase e peroxidase utilizando eletroforese em géis de poliacrilamida. A enzima peroxidase foi a única eficiente no monitoramento da deterioração e perda da capacidade germinativa de sementes de palmiteiro-vermelho.
\end{abstract}

Palavras-chave: Palmeira, Recalcitrante e Peroxidase.

\section{ISOENZYMES FOR MONITORING DETERIORATION OF Euterpe espiritosantensis FERNANDES SEEDS}

\begin{abstract}
Red-palmito Euterpe espiritosantensis Fernandes seeds are recalcitrants because they exhibit germination decreases by dehydration and have short longevity. The objective of this work was to identify efficient enzymatic systems to monitoring deterioration and loss of germination capacity of red-palmito seeds. Seeds were dryed during 0, 20, and 40h (resulting moisture content of 46, 40 and 36\%, respectively) and stored at $15^{\circ} \mathrm{C}$, inside closed plastic bags, during 54 weeks. The seed quality was evaluated, in six weeks intervals, by germination test and by enzymatic activity of glucose-6-phosphate dehydrogenase, glutamate dehydrogenase, phosphoglucomutase, phosphoglucose isomerase and peroxidase, using the electrophoresis technique in polyacrylamide gel. The isoenzyme peroxidase was the unique efficient way to monitor deterioration and loss of germination capacity of red-palmito seeds.
\end{abstract}

Key words: Palm, Recalcitrant Seedseed and Peroxidase.

\section{INTRODUÇÃO}

O palmiteiro-vermelho (Euterpe espiritosantensis Fernandes) é uma espécie nativa da Floresta Tropical Atlântica, cuja propagação natural se dá exclusivamente por sementes. Formações florestais naturais dessa planta foram encontradas na região de Santa Teresa, no Estado do Espírito Santo (FERNANDES, 1989), em Ituberá e Itabuna, no Estado da Bahia (BOVI et al., 1987 e 1994). A crescente devastação do seu habitat, associada à derrubada de plantas para a extração de palmito, torna de interesse a produção de sementes e mudas dessa espécie para a recomposição da flora nativa. Conhecimentos sobre a fisiologia da semente e da germinação são necessários para o desenvolvimento de tecnologias favoráveis à produção à conservação das sementes dessa espécie.

As sementes do palmiteiro-vermelho foram classificadas como recalcitrantes devido à sensibilidade à desidratação e à curta longevidade no armazenamento (MARTINS et al., 1999a; MARTINS et al., 2007ab). A

\footnotetext{
${ }^{1}$ Recebido em 30.06.2008 e aceito para publicação em 25.08.2010.

${ }^{2}$ Faculdade de Tecnologia do Estado de São Paulo - Campus de Capão Bonito, FATEC, Brasil. E-mail: <cibele@fca.unesp.br>. ${ }^{3}$ Universidade Estadual Paulista Júlio de Mesquita Filho, UNESP, Brasil. E-mail: <damv@fca.unesp.br>.

${ }^{4}$ Departamento de Biofísica, Instituto de Biociências, Unesp, Botucatu, SP.
} 
perda de água em sementes recalcitrantes desencadeia inúmeros processos deteriorativos, como a desnaturação de proteínas, alterações na atividade das enzimas e danos no sistema de membranas, resultando, finalmente, na completa perda de viabilidade das sementes (NAUTIYAL e PUROHIT, 1985).

A incapacidade dos tecidos das sementes sensíveis à dessecação em efetuar adequada proteção contra eventos oxidativos, consequentes do metabolismo desorganizado durante a desidratação e o armazenamento, pode ser considerada como uma das principais causas da sensibilidade à dessecação e da reduzida longevidade (LEPRINCE et al., 1993). Isso porque o balanço entre a geração e remoção de radicais, durante a secagem e armazenamento das sementes, relaciona-se com a sua longevidade. Danos celulares causados pela peroxidação de lipídios podem ser reduzidos ou prevenidos por mecanismos protetores, entre os quais se podem citar os provenientes das ações de enzimas removedoras de radicais livres e de peróxidos, como a peroxidase (MENEZES, 2005). A atividade da glucose-6-fosfato desidrogenase, enzima pertencente à rota das pentoses, é importante na determinação do fluxo através da glicólise. O balanço entre a glicólise e a rota das pentoses assegura suprimento adequado às sementes de poder redutor, ATP e esqueletos de carbono para a biossíntese de macromoléculas. Assim, a atividade dessa enzima é minimizada pela deterioração(BETTEY e FINCH-SAVAGE, 1996). As isomerases catalisam a conversão da glicose em frutose durante a germinação das sementes, e a glutamato desidrogenase atua na oxidação de proteínas de reserva, fornecendo energia para as células e, ou, na redução da cetoglutarato para a síntese de aminoácidos (BRANDÃO-JÚNIOR et al., 1999).

Apesar de existir considerável informação sobre alterações em enzimas específicas relacionadas à deterioração de sementes ortodoxas, essa área do conhecimento ainda foi pouco explorada em sementes recalcitrantes. Assim, trabalhos de monitoramento da deterioração utilizando a técnica de eletroforese com a isoenzima peroxidase têm apresentado resultados promissores para sementes de milho(BRANDÃO JUNIOR et al., 1999; SPÍNOLA et al., 2000), algodão (VIEIRA, 1996) e copaíba (CARVALHO et al., 2006). Para sementes de milho e copaíba submetidas a envelhecimento acelerado, verificou-se diminuição no número e intensidade das bandas da peroxidase, com o aumento da deterioração e perda da capacidade germinativa (BRANDÃO JUNIOR et al., 1999; CARVALHO et al., 2006).

Revista Árvore, Viçosa-MG, v.35, n.1, p.85-90, 2011
Para a aplicação da técnica de eletroforese de isoenzimas de forma satisfatória, deve-se considerar a adequação da metodologia à espécie em estudo e ao tipo e idade do material vegetal das amostras, pois esses fatores afetam a expressão, resolução e estabilidade das bandas isoenzimáticas nos géis (BALLVÉ, 1988). Para sementes de palmiteiro-vermelho, Martins et al. (2007b) verificaram que a técnica utilizada permitiu perfeita corrida eletroforética e resolução dos perfis para as isoenzimas glucose-6-fosfato desidrogenase, glutamato desidrogenase, fosfoglucomutase, fosfoglucose isomerase e peroxidase.

Assim, o objetivo deste trabalho foi identificar enzimas eficientes no monitoramento da deterioração e perda da capacidade germinativa de sementes de palmiteiro-vermelho.

\section{MATERIAL E MÉTODOS}

Frutos maduros foram colhidos na coleção de palmeiras do Instituto Agronômico (Ubatuba/SP) em 17 plantas-matriz, em agosto de 1999. As sementes malformadas, danificadas ou abaixo do tamanho médio foram eliminadas.

As sementes foram colocadas para secar por 0 , 20 e 40 h (três sublotes) em câmara seca (temperatura de $22^{\circ} \mathrm{C}$ e umidade relativa de $38 \%$ ), visando à obtenção de teores distintos de água nos três sublotes. Após a secagem, os três sublotes foram armazenados no escuro a $15^{\circ} \mathrm{C}$, em sacos plásticos (20 ìm de espessura) fechados (MARTINS et al., 2000) por 54 semanas, e em intervalos de tempo de seis semanas foram realizadas as seguintes determinações em laboratório.

Teor de água: avaliado pelo método da estufa a $105 \pm 3^{\circ} \mathrm{C} / 24 \mathrm{~h}$ (BRASIL, 1992), utilizando-se quatro repetições de sete sementes inteiras. Germinação: conduzido com quatro repetições de 20 sementes, mantidas nas temperaturas alternadas de $20-30^{\circ} \mathrm{C}$ e luz (8 h), em substrato vermiculita, esterilizada $\left(120^{\circ} \mathrm{C} / 12 \mathrm{~h}\right)$, em caixas plásticas transparentes ( 11 x 11x 3,5 cm) (BRASIL, 1992; MARTINS et al., 2007a). A contagem das plântulas foi realizada semanalmente, do sétimo ao $49^{\circ}$ dia após a semeadura, quando foi calculada a porcentagem de germinação (plântulas normais).

Atividade dos sistemas enzimáticos As sementes foram postas para germinar nas mesmas condições descritas no teste de germinação. Botões germinativos recém-emitidos (MARTINS et al., 1999b) 
com 0 a $1 \mathrm{~mm}$ de protrusão e obtidos três a sete dias após a semeadura foram extraídos com o auxílio de um bisturi e submetidos à análise eletroforética. Nas análises de eletroforese de isoenzimas foram utilizados 30 embriões por tratamento. Assim, realizaram-se as comparações dos graus de polimorfismo de cinco sistemas enzimáticos: glucose-6-fosfato desidrogenase (G6PDH), glutamato desidrogenase (GDH), fosfoglucomutase (PGM), fosfoglucose isomerase (PGI) e peroxidase (PO), em géis de poliacrilamida, em um número mínimo de quatro corridas $\left(3 \pm 1^{\circ} \mathrm{C}\right)$ por tratamento e enzima avaliada. A obtenção das amostras, o preparo das soluções para cada sistema enzimático e os procedimentos de corrida e coloração utilizados seguiram a metodologia descrita por Martins et al. (2007b) para sementes de palmiteiro-vermelho. Para a identificação das bandas foi calculado o coeficiente de mobilidade relativa (Rf), que representa a relação entre a distância de migração da isoenzima (I) e a distância de migração do corante (Fm), isto é, $\mathrm{Rf}=\mathrm{I} / \mathrm{Fm}$.

Os perfis eletroforéticos de sistemas enzimáticos que apresentaram alterações na intensidade de cor e bandas discriminantes correlacionadas com deterioração das sementes tiveram esses parâmetros quantificados em quatro repetições de nove perfis eletroforéticos de cada tratamento, através do programa Eagle-eye de análise de géis (Stratagene), tendo-se o cuidado de descontar nesta análise a coloração de fundo de cada gel. Os resultados foram apresentados em pixels, que é a quantidade de pigmentação média por $\mathrm{mm}^{2}$ da imagem do gel.

A análise de variância foi realizada separadamente para cada teste, em delineamento inteiramente casualizado com quatro repetições, em arranjo fatorial $3 \times 10$, em que três foram os tempos de secagem e 10, os tempos de armazenamento. A comparação entre as médias dos tratamentos foi efetuada através do teste de Tukey a 5\% de probabilidade. Os dados expressos em porcentagem foram transformados em arcosseno $(\mathrm{x} / 100)^{1 / 2}$, e quando ocorreu valor igual a zero os dados em porcentagem foram transformados em $(x+0,5)^{1 / 2}$.

\section{RESULTADOS E DISCUSSÃO}

Na Tabela 1 estão apresentadas as médias comparadas pelo teste de Tukey. O aumento do tempo de secagem resultou em sementes com teores de água decrescentes e que se mantiveram praticamente inalterados durante o armazenamento, com médias de 46, 40 e 36\% para 0, 20 e $40 \mathrm{~h}$ de secagem, respectivamente. A germinação do lote não foi afetada pela secagem, somente nas sementes sem armazenamento, apresentando 89, 92 e 89\% de germinação para 0, 20 e 40 h de secagem, respectivamente. Houve perda total da viabilidade por volta da $30^{\mathrm{a}}$ semana apenas no sublote de $40 \mathrm{~h}$ de secagem (teor de água de 36\%), enquanto os demais ainda se apresentavam viáveis até 54 semanas de armazenamento (média de 46\% de germinação).

Dos sistemas enzimáticos testados, a glutamato desidrogenase (GDH), a glucose-6-fosfato desidrogenase (G6PDH), a fosfoglucomutase (PGM) e a fosfoglucose isomerase (PGI) apresentaram todas as bandas nítidas e sem alterações de posição e intensidade de cor que poderiam ser devidas à secagem e ao armazenamento em todas as corridas. Desse modo, embora existam relatos de que algumas dessas enzimas poderiam ser afetadas pelo processo de envelhecimento dos tecidos (BETTEY e FINCH-SAVAGE, 1996; BRANDÃO-JÚNIOR et al., 1999), esses sistemas enzimáticos não foram eficientes na avaliação da deterioração das sementes recalcitrantes de palmiteiro-vermelho. Isso provavelmente ocorreu porque a atividade enzimática depende da espécie vegetal, do tipo e idade da estrutura da planta da qual são obtidas as amostras (BALLVÉ, 1988; MARTINS et al., 2007b). Os perfis eletroforéticos obtidos com essas isoenzimas foram idênticos aos relatados por Martins et al. (2007b) para sementes de palmiteiro-vermelho.

Somente a peroxidase (PO) apresentou eficiência na identificação da deterioração de sementes de $E$. espiritosantensis, pois os perfis eletroforéticos (Figuras 1 e 2) apresentaram a última banda, localizada na parte inferior do gel (assinalada com seta) e situada na posição $\mathrm{Rf}=$ 0,38 (MARTINS et al., 2007b), menos intensa quanto menor a germinação das sementes. Por isso, essa banda foi identificada como discriminante e correlacionada com a deterioração das sementes e pôde ser utilizada como referência de vigor mediante a quantificação da intensidade de sua cor nos perfis eletroforéticos da peroxidase. Assim, o decréscimo do teor de água e o aumento do tempo de armazenamento prejudicaram a atividade da enzima peroxidase. Esse fato pode ser explicado pelas características recalcitrantes das sementes de E. espiritosantensis, de sensibilidade à desidratação e problemas de conservação pós-maturidade fisiológica (MARTINS et al., 1999a).

A análise estatística da intensidade da última banda da peroxidase (Tabela 1) demonstra que não houve efeito prejudicial da secagem das sementes sobre a atividade da peroxidase até 18 semanas de armazenamento. Foi verificada redução significativa da intensidade da banda após 48 semanas de armazenamento das sementes secadas por $20 \mathrm{~h}$ (teor de água de 41\%) e após 24 semanas daquelas secadas por 40 h (teor de água de 36\%).

Revista Árvore, Viçosa-MG, v.35, n.1, p.85-90, 2011 
Tabela 1 - Médias das características utilizadas na avaliação da qualidade fisiológica das sementes de Euterpe espiritosantensis submetidas a três tempos de secagem e armazenadas durante 54 semanas.

Table 1 - Means of the characteristics to evaluate physiological quality of Euterpe espiritosantensis seeds submitted to three times of drying and stored during 54 weeks.

\begin{tabular}{|c|c|c|c|c|}
\hline \multirow[t]{2}{*}{ Características avaliadas } & \multirow{2}{*}{$\begin{array}{l}\text { Armazenamento } \\
\text { (semanas) }\end{array}$} & \multicolumn{3}{|c|}{ Tempo de secagem (horas) } \\
\hline & & 0 & 20 & 40 \\
\hline \multirow{3}{*}{ Teor de água (\%) } & 0 & 45,19 a $A^{*}$ & 39,25 b B & 35,14 bc $C$ \\
\hline & 6 & 45,20 a $\mathrm{A}$ & 39,27 b B & 34,77 с C \\
\hline & 12 & 46,52 a $\mathrm{A}$ & 38,88 b B & 35,62 abc $C$ \\
\hline \multirow[t]{8}{*}{$\mathrm{CV} \%=10,7$} & 18 & 46,89 а $\mathrm{A}$ & 38,71 b B & 35,20 bc $\mathrm{C}$ \\
\hline & 24 & 46,06 а A & 40,99 ab B & 36,39 ab $\mathrm{C}$ \\
\hline & 30 & 45,59 а A & $40,04 \mathrm{ab} B$ & 36,33 ab $\mathrm{C}$ \\
\hline & 36 & 45,88 а A & 39,68 ab B & 36,29 ab $\mathrm{C}$ \\
\hline & 42 & 47,06 а A & 40,82 ab B & 36,61 a $C$ \\
\hline & 48 & 46,27 а A & 41,15 ab B & 36,22 ab $C$ \\
\hline & 54 & 45,55 а $\mathrm{A}$ & 42,11 a $\mathrm{A}$ & 36,08 abc B \\
\hline & 0 & 88,75 ab A & 92,50 a $\mathrm{A}$ & 88,75 a $A$ \\
\hline \multirow[t]{3}{*}{ Germinação (\%) } & 6 & 98,75 a $\mathrm{A}$ & 87,50 ab $\mathrm{AB}$ & 81,25 ab $\mathrm{B}$ \\
\hline & 12 & 91,25 ab A & 81,25 abc $\mathrm{AB}$ & $75,00 \mathrm{~b} \mathrm{~B}$ \\
\hline & 18 & 88,75 ab A & 82,50 abc $\mathrm{AB}$ & 72,50 b B \\
\hline \multirow[t]{6}{*}{$\mathrm{CV} \%=52,7$} & 24 & 85,00 abc $\mathrm{A}$ & 87,50 ab A & 20,00 с В \\
\hline & 30 & 81,25 abc $\mathrm{A}$ & 68,75 bcd A & $0,00 \mathrm{~d} B$ \\
\hline & 36 & 86,25 ab A & $61,25 \mathrm{~cd} \mathrm{~B}$ & $10,00 \mathrm{~cd} \mathrm{C}$ \\
\hline & 42 & 70,00 bc $A$ & $57,50 \mathrm{~d} A$ & $0,00 \mathrm{~d} B$ \\
\hline & 48 & 61,25 cd A & $48,75 \mathrm{~d} \mathrm{~A}$ & $0,00 \mathrm{~d} B$ \\
\hline & 54 & $43,75 \mathrm{~d} \mathrm{~A}$ & $48,75 \mathrm{~d} \mathrm{~A}$ & $0,00 \mathrm{~d} \mathrm{~B}$ \\
\hline Peroxidase & 0 & 65,00 а A & 67,00 a $\mathrm{A}$ & 65,00 a $A$ \\
\hline \multirow{4}{*}{$\begin{array}{c}\text { Banda } \mathrm{Rf}=0,38 \\
\text { (pixels) }\end{array}$} & 6 & 67,75 а A & 62,75 a $A$ & 57,25 a $\mathrm{A}$ \\
\hline & 12 & 65,50 а $\mathrm{A}$ & 58,00 a $A$ & 59,75 a $\mathrm{A}$ \\
\hline & 18 & 63,50 а $A$ & 56,00 a $A$ & 56,75 a $A$ \\
\hline & 24 & 61,75 а A & 61,50 a $A$ & $10,00 \mathrm{~b} \mathrm{~B}$ \\
\hline \multirow[t]{5}{*}{$\mathrm{CV} \%=54,4$} & 30 & 61,25 а A & 62,50 a $A$ & $0,00 \mathrm{~b} \mathrm{~B}$ \\
\hline & 36 & 62,25 а A & 57,75 a $\mathrm{A}$ & $0,00 \mathrm{~b} \mathrm{~B}$ \\
\hline & 42 & 62,50 а $A$ & 60,25 a $A$ & $0,00 \mathrm{~b} \mathrm{~B}$ \\
\hline & 48 & 56,75 а A & 31,50 b B & $0,00 \mathrm{~b} \mathrm{C}$ \\
\hline & 54 & 34,75 а A & 25,75 b B & $0,00 \mathrm{~b} \mathrm{C}$ \\
\hline
\end{tabular}

* Para cada característica avaliada, médias seguidas pela mesma letra minúscula na coluna e maiúscula na linha não diferem estatisticamente entre si, pelo teste de Tukey $(P<0,05)$.

Os perfis eletroforéticos da isoenzima peroxidase (PO) para botões germinativos de E. espiritosantensis retirados de sementes secadas por $40 \mathrm{~h}$ (teor de água de $35 \%$ ) e com tempo de armazenamento, germinação e intensidade de coloração para a última banda de, respectivamente, 0 semana, 89\% e 65 pixels (a) e 36 semanas, $10 \%$ e zero pixel (b), respectivamente, estão apresentados na Figura 1.

A diminuição do número e, ou, da intensidade de bandas da peroxidase (PO) com o aumento da deterioração está de acordo com os resultados obtidos por Nkang (1988), Basavarajappa et al. (1991), Jeng e Sung (1994), Brandão Junior et al. (1999), Vieira (1996),
Spínola et al. (2000) e Carvalho et al. (2006). Isso pode ser explicado pelo fato de a peroxidase ser uma enzima removedora de peróxido, e a perda de sua atividade pode tornar a semente mais sensível aos efeitos de $\mathrm{O}_{2}$ e radicais livres sobre ácidos graxos insaturados de membrana, o que provoca a degeneração dessas e o comprometimento do vigor.

O perfil eletroforético da isoenzima peroxidase (PO) para botões germinativos de E. espiritosantensis retirados de sementes armazenadas por 36 semanas e com teor de água, germinação e intensidade de coloração de, respectivamente, $46 \%$, 86\% e 62 pixels (a), 40\%, 61\% e 58 pixels (b), 36\%, 10\% e zero pixel

Revista Árvore, Viçosa-MG, v.35, n.1, p.85-90, 2011 
(c) está apresentado na Figura 2, demonstrando o desaparecimento da última banda com o aumento do tempo de armazenamento e deterioração; as demais bandas não demonstraram alteração de intensidade. Esses resultados ratificam as indicações de que a banda na posição $\mathrm{Rf}=0,38$ seria discriminativa para a deterioração de sementes dessa espécie. Para Vieira (1996), a perda

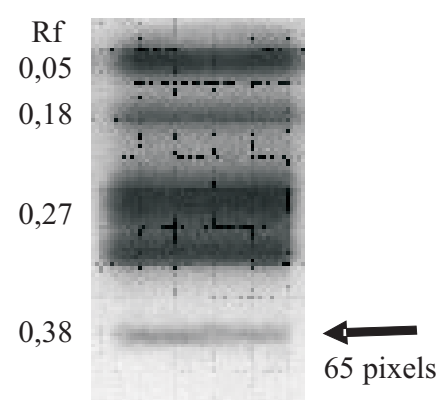

a

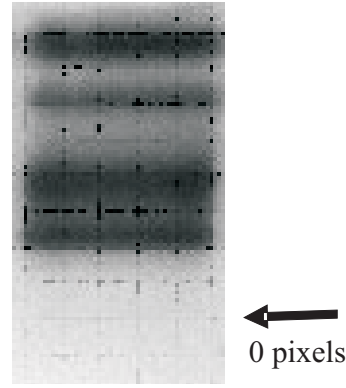

b
Figura 1 - Perfis eletroforéticos da isoenzima peroxidase (PO) de botões germinativos de Euterpe espiritosantensis retirados de sementes secadas por $40 \mathrm{~h}$ (teor de água de 35\%) e com tempo de armazenamento de 0 (a) e 36 semanas (b).

Figure 1 -Electrophoretic profile of peroxidase isoenzyme (PO) for germinative button removed from Euterpe espiritosantensis seeds dryed during 40h (35\% of moisture content), and with storage time and, of 0 (a) and 36 weeks (b).

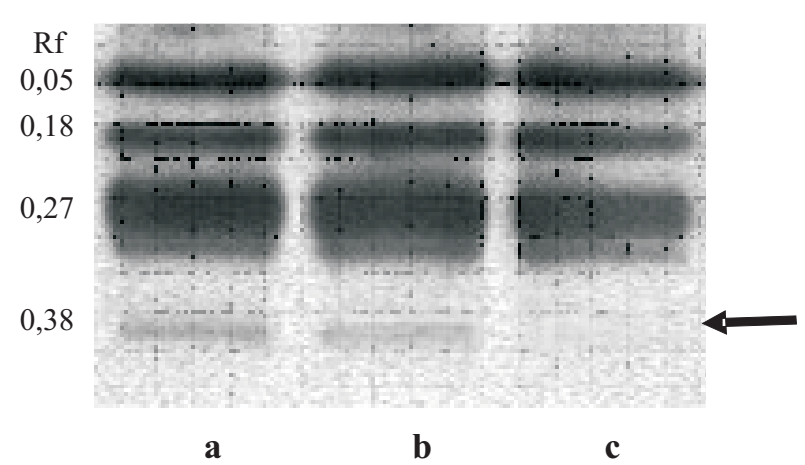

Figura 2 - Perfis eletroforéticos da isoenzima peroxidase (PO) de botões germinativos de Euterpe espiritosantensis retirados de sementes submetidas à secagem por 0 (a), 20 (b) e 40 h (c) e armazenadas por 36 semanas.

Figure 2-Electrophoretic profile of peroxidase isoenzyme (PO) for germinative button removed from Euterpe espiritosantensis seeds dryed during 0 (a), 20 (b), and $40 \mathrm{~h}(\mathrm{c})$, and stored during 36 weeks. completa da atividade da peroxidase, durante o envelhecimento, pode esclarecer parcialmente o acúmulo de peróxidos em sementes envelhecidas.

Assim, pôde-se concluir que a enzima peroxidase foi a única eficiente no monitoramento da deterioração e perda da capacidade germinativa de sementes de palmiteiro-vermelho.

\section{AGRADECIMENTOS}

À FAPESP (Programa Jovem Pesquisador), pelo financiamento deste trabalho.

\section{REFERÊNCIAS}

BALLVÉ, R. M. L. Isoenzimas como marcadores genéticos em palmiteiro (Euterpe spp.). 1988. 86f. Dissertação (Mestrado em Biologia) - Universidade Estadual de Campinas, Campinas, 1988.

BASAVARAJAPPA, B. S.; SHETTY, H. S.; PRAKASH, H. S. Membrane deterioration and other biochemical changes, associated with accelerated ageing of maize seeds. Seed Science and Technology, v.19, n.2, p.279-286, 1991.

BETTEY, M.; FINCH-SAVAGE, W. E. Respiratory enzyme activities during germination in Brassica seed lots of differing vigour. Seed Science Research, v.6, p.165-173, 1996.

BOVI, M.L.A.; GODOY-JÚNIOR, G.; SAES, L.A. Pesquisas com os gêneros Euterpe e Bactris no Instituto Agronômico de Campinas.

Agronômico, v.39, n.2, p.129-174, 1987.

BOVI, M. L. A. et al. Floral biology and reproductive system of Euterpe espiritosantensis Fernandes. Acta Horticulturae, v.360, n.8, p.41-56, 1994.

BRANDÃO JUNIOR, D. S.; CARVALHO, M. L. M.; VIEIRA, M. G. G. C. Variações eletroforéticas de proteínas e isoenzimas relativas à deterioração de sementes de milho envelhecidas artificialmente Revista Brasileira de Sementes, v. 21, n.1, p.114-121, 1999.

BRASIL. MINISTÉRIODAAGRICULTURAE REFORMA AGRARIA. Regras para análise de sementes. Brasília: SNDA/DNDV/CLAV, 1992. 365p.

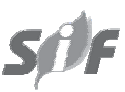

Revista Árvore, Viçosa-MG, v.35, n.1, p.85-90, 2011 
CARVALHO, D. et al. Eletroforese de proteínas e isoenzimas em sementes de Copaifera langsdorffii Desf. (leguminosae caesalpinioideae) envelhecidas artificialmente. Revista Árvore, v.30, n.1, p.19-24, 2006.

FERNANDES, H. Q. B. Uma nova espécie de Euterpe (Palmae - Arecoideae - Areceae) do Brasil. Acta Botânica Brasílica, v.3, n.1, p.43-49, 1989.

JENG, T. L.; SUNG, J. M. Hydration effect on lipid peroxidation and peroxide-scavening enzymes activity of artificially age peanut seed. Seed Science and Technology, v.22, n.3, p.531-539, 1994.

LEPRINCE, O.; HENDRY, G. A. F.; MCKERSIE, B. $D$. The mechanisms of desiccation tolerance in developing seeds. Seed Science Research, v.3, n.2, p.231-246, 1993.

MARTINS C. C.; NAKAGAWA, J.; BOVI, M. L. A. Tolerância à dessecação de sementes de palmiteiro-vermelho (Euterpe espiritosantensis Fernandes). Revista Brasileira de Botânica, v.22, n.3, p.391-396, 1999a.

MARTINS, C. C.; NAKAGAWA, J.; BOVI, M. L. A. Dessication tolerance of four seeds lots of Euterpe edulis Mart. Seed Science and Technology, v.28, n.1, p.1-13, 1999b.

MARTINS, C. C. et al. Despolpamento e temperatura no armazenamento temporário de sementes de palmiteiro-vermelho (Euterpe espiritosantensis Fernandes). Revista Brasileira de Sementes, v.22, n.1, p.169-176, 2000.
MARTINS, C. C.; BOVI, M. L. A.; NAKAGAWA, J. Qualidade fisiológica de sementes de palmiteirovermelho em função da desidratação e do armazenamento. Horticultura Brasileira, v.25, n. 2, p.188-192, 2007a.

MARTINS, C. C. et al. Isoenzimas na diferenciação de sementes de três espécies do gênero Euterpe. Revista Árvore, v.31, n.1, p.51-57, 2007b.

MENEZES, M. Identificação de cultivares de milho, feijão, algodão e soja por meio de enzimas e de proteínas resistentes ao calor. 2005. 91f. Dissertação (Mestrado em Fitotecnia) - Universidade Federal de Lavras, Lavras, 2005.

NAUTIYAL, A.R.; PUROHIT, A.N. Seed viability in sal. II. Physiological and biochemical aspects of ageing in seeds of Shorea robusta. Seed Science and Technology, v.13, n.1, p.69-76, 1985.

NKANG, A. Some aspects of the biochemical basis of viability of loss in stored Guilfoylia monostylis seeds. Seed Science and Technology, v.16, n.1, p.247-260, 1988.

SPINOLA, M. C. M.; CÍCERO, S. M.; MELO, M. Alterações bioquímicas e fisiológicas em sementes de milho causadas pelo envelhecimento acelerado. Scientia Agrícola, v.57, n.2, p.263-270, 2000.

VIEIRA, M. G. C. G. Utilização de marcadores moleculares no monitoramento da qualidade sanitária e nível de deterioração de sementes de algodoeiro (Gossypium hirsutum L.). 1996. 127f. Tese (Doutorado em Fitotecnia) Universidade Federal de Lavras, Lavras, 1996. 